\title{
Missed aortic valve endocarditis resulting in complete atrioventricular block and redo aortic valve replacement
}

\author{
Amer Harky MRCS ${ }^{1}$, Miruna Popa ${ }^{2}$, Megan Garner ${ }^{1}$, Alex Shipolini ${ }^{1}$
}

Harky A, Popa M, Garner M. Missed aortic valve endocarditis resulting in complete atrioventricular block and redo aortic valve replacement. Curr Res Cardiol 2017;4(2):22-23

\section{ABSTRACT}

BACKGROUND: Infective endocarditis is a rare disease associated with high morbidity and mortality. As a result, early diagnosis, prompt antibiotic treatment with or without surgical intervention is crucial in the management of such a condition.

CASE REPORT: We report a case of missed infective endocarditis of the aortic valve. The patient underwent mechanical aortic valve replacement, with the native valve sent for histopathological analysis. On re-admission with syncope and shortness of breath he was noted to have complete heart block and severe aortic regurgitation and paravalvular leak. DISCUSSION: On review of the histopathology, endocarditis was identified which had not been acted upon at the original operation. The patient underwent redo aortic valve replacement with insertion of a permanent pacemaker and treatment with appropriate antibiotics.

CONCLUSION: We highlight the importance of following up histopathological results as well as the need for multidisciplinary treatment of endocarditis with a combination of surgical and antibiotic therapy.

Key Words: Infective endocarditis; Aortic valve surgery; Redo sternotomy
A 52-year-old male presented to his GP with shortness of breathing on Iexertion (NYHA III), fatigue, dizziness and significant weight loss of 10 kilograms over a three months period. His past medical history included cholecystectomy, a tooth extraction during the preceding year and he was a current smoker. Clinical examination revealed a diastolic murmur; subsequent transthoracic echocardiography (TTE) demonstrated severe aortic regurgitation.

Elective mechanical aortic valve replacement using a size $23 \mathrm{~mm}$ Sorin Carbomedics $^{\circledR}$ valve was performed. Intraoperative findings revealed a tricuspid aortic valve, however the non-coronary cusp was virtually absent due to a severe destructive process. The left coronary cusp was thickened especially at the annulus. Both remaining aortic valve leaflets were sent for histopathological analysis. The patient had an uncomplicated postoperative course and he was discharged on day nine postoperatively with lifelong warfarin therapy.

Thirteen months following his surgery, the patient reported to his local hospital with persistent fatigue and increasing shortness of breathing on exertion (NYHA III). This had prevented him from completing his cardiac rehabilitation program and resuming his previous work. Retrospectively, he reported severe night sweats since his aortic valve replacement. Clinical examination was satisfactory at this stage and he was treated with a short course of antibiotics for assumed lower respiratory tract infection.

Three months later he presented to the emergency unit with repeated shortness of breathing and multiple syncopal episodes. He was found to be in complete heart block and an emergency permanent pacemaker was inserted. Post pacemaker insertion TTE revealed "rocking" aortic valve prosthesis with severe aortic regurgitation in parallel with significant paraprosthetic and central leak (Figure 1, Video 1). This admission was further complicated by septic shock requiring admission to intensive care for intravenous antibiotics and haemofiltration. He underwent emergency redo sternotomy and aortic valve replacement with a size $25 \mathrm{~mm}$ Sorin carbomedics ${ }^{\circledR}$ mechanical prosthesis. Intra-operative findings were circumferential valve dehiscence and significant annular destruction especially around the non-coronary area. No aortic root abscess or vegetations were found. Postoperatively, he was transferred in stable condition to the intensive care unit and commenced on intravenous antibiotics for infective endocarditis as per hospital protocol (Vancomycin, Gentamicin and Rifampicin).

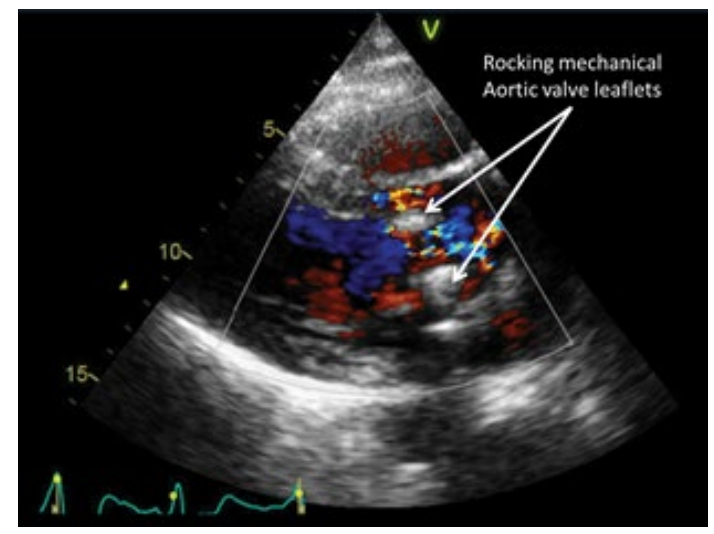

Figure 1) Transthoracic echocardiography - short axis view showing the rocking aortic valve

Whilst the patient was in intensive care it was noted that the histopathology report from the first operation had identified endocarditis as the cause of the severe aortic regurgitation but this had not been acted upon on his initial surgical admission. The histopathology report of the prosthetic valve explant was negative for leucocytes and microorganisms; in addition, PCR analysis of aortic annular tissue did not detect bacterial DNA. All subsequent blood cultures remained negative. A diagnosis of culture-negative infective endocarditis was made and the patient completed the appropriate course of antibiotics. His postoperative TTE showed a well-seated aortic valve (Figure

${ }^{1}$ Department of Cardiac Surgery, Barts Heart Centre, St. Bartholomew's Hospital, London, UK, ${ }^{2}$ Heidelberg medical faculty, Heidelberg University, Germany.

Correspondence: Dr Amer Harky, MRCS, Department of Cardiac Surgery, Barts Heart Centre, St. Bartholomew's Hospital, London, UK, Telephone +44 2034165000 , E-mailaaharky@gmail.com

Received: May 04, 2017, Accepted: July 06, 2017, Published: July 08, 2017 
2, Video 2). At the patients six months follow up appointment, he is doing well and denies any cardiac symptoms.

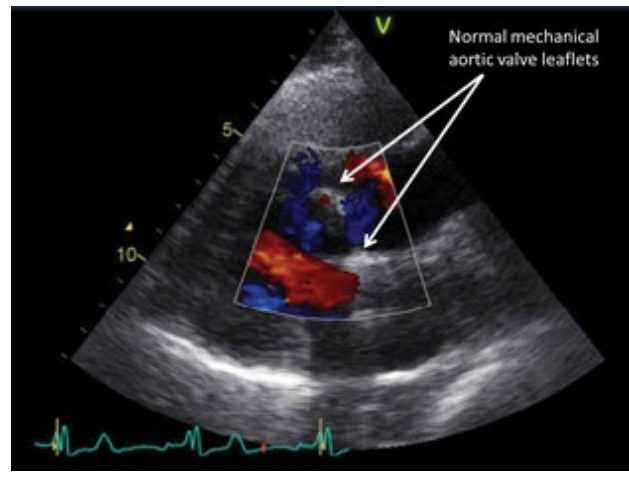

Figure 2) Post re-do surgery transthoracic echocardiography - short axis view showing normally functioning aortic valve

\section{DISCUSSION}

Infective Endocarditis (IE) is an infection of endocardium or cardiac valves. Prior to the introduction of current aggressive antibiotic treatment regimens, the mortality rate from this condition was very high $(1,2)$. Early diagnosis and prompt initiation of antibiotic therapy are essential in the treatment of IE, and delayed diagnosis is unfortunately not uncommon. Furthermore, previous research has shown that $25 \%$ of IE patients are admitted to hospital more than one month after their symptom onset, which is thought to be due to the large range of indistinct symptoms associated with IE (3). Missed endocarditis after surgery is rather uncommon since valve histology is an indispensable diagnostic tool $(4,5)$. Re-presentation of IE, especially of the aortic valve, can be life threatening as it may present with septic episodes as well as acute kidney injury $(6-30 \%)$ and conduction abnormalities (1-15\%) (6).

In our case although the operative findings were suggestive of IE, the clinical presentation was atypical. The majority of patients with IE present with fever, however our patient remained apyrexial during the entire disease course. In addition, typical vegetation's were absent both in the echocardiography and in the surgical specimen. As a result, the clinical modified Duke criteria necessary for the diagnosis of IE were not fully met (7). Despite this atypical presentation, suspicions should have arisen with regard to the patient's unspecific symptoms of fatigue, shortness of breath, loss of appetite, weight loss. Furthermore, the histopathological report showing clear features of IE was missed during the first surgical admission and at follow-up.

There are very few previous reports on missed endocarditis following surgical valve replacements. Ferraz et al. (8) described a case of culture-negative endocarditis resulting in recurrent mechanical valve dehiscence in the mitral position. After the third episode requiring surgical intervention, serology revealed a Coxiella burnetii infection, which had persisted as chronic $Q$ fever for years. The present case and the report of Ferraz et al. (8) both highlights that culture-negative endocarditis can be particularly challenging to diagnose and therefore treatment should not be delayed while awaiting culture results. Since culture-negative endocarditis is known to occur in up to $31 \%$ of cases (9), it is of paramount consider this as a differential diagnosis especially in patients with prosthetic valve replacement presenting with the non-specific symptoms suggestive of infection.

\section{CONCLUSION}

In conclusion, surgery by its self cannot guarantee complete resolution of infective endocarditis. Antibiotic treatment is therefore indispensable in its management for total remission. This case highlights the importance of effective communication between pathologists, microbiologists and cardiothoracic surgeons to ensure the appropriate treatment is commenced. Finally, an intraoperative finding of destructive valve disease should prompt to an immediate histopathological examination of the diseased valve, antibiotic therapy should not be delayed whilst awaiting these results.

\section{REFERENCES}

1. Verhagen DWM, Vedder AC, Speelman P, et al. Anti-microbial treatment of infective endocarditis caused by viridans Streptococci highly susceptible to penicillin: A historic overview and future considerations. J Antimicrob Chemother 2006;57:819-24.

2. Larry MB, et al. Infective endocarditis in adults: Diagnosis, antimicrobial therapy, and management of complications, Circulation 2015;132.

3. Celik M, Yuksel U, Yalcinkaya E, et al. Conservative treatment of iatrogenic left main coronary artery dissection: A report of two cases. Cardiovasc Diagn Ther 2013;3:244-46.

4. Hubert L, Jean-Paul C, Pierre-Edouard F, et al. Quantitative histological examination of mechanical heart valves. Clin Infect Dis 2005;40:655-61.

5. Brandão TJ, Januario-da-Silva CA, Correia MG, et al. Histopathology of valves in infective endocarditis, diagnostic criteria and treatment considerations. Infection. 2017;45:199-07.

6. Habib G, Hoen B, Tornos P, et al. Guidelines on the prevention, diagnosis, and treatment of infective endocarditis. Eur Heart J 2009.

7. Durack DT, Lukes AS, Bright DK. New criteria for diagnosis of infective endocarditis: utilization of specific echocardiographic findings. Duke Endocarditis Service. Am J Med 1994;96:200-09

8. Ferraz RV, Andrade M, Silva F, et al. Chronic Q fever: A missed prosthetic valve endocarditis possibly for years. ID Cases. 2016;6:55-7.

9. Brouqui P, Raoult D. Endocarditis due to rare and fastidious bacteria. Clinical Microbiology Reviews 2001;14:177-07 